\title{
Performance of the Dark Energy Camera Liquid Nitrogen Cooling System
}

\author{
H. Cease*, M. Alvarez*, R. Alvarez ${ }^{\dagger}$, M. Bonati ${ }^{\dagger}$, G. Derylo*, J. Estrada*, B. \\ Flaugher*, R. Flores*, A. Lathrop*, F. Munoz ${ }^{\dagger}$, R. Schmidt ${ }^{\dagger}$, R. L. Schmitt*, K. \\ Schultz $^{*}$, S. Kuhlmann ${ }^{* *}$ and A. Zhao** \\ *Fermi National Accelerator Laboratory, Batavia, Illinois 60510, USA \\ ${ }^{\dagger}$ Cerro Telolo Inter-American Observatory, La Serena, Chile \\ ${ }^{* *}$ Argonne National Laborator, Argonne, Illinois 60439, USA
}

\begin{abstract}
The Dark Energy Camera, the Imager and its cooling system was installed onto the Blanco 4m telescope at the Cerro Tololo Inter-American Observatory in Chile in September 2012. The imager cooling system is a LN2 two-phase closed loop cryogenic cooling system. The cryogenic circulation processing is located off the telescope. Liquid nitrogen vacuum jacketed transfer lines are run up the outside of the telescope truss tubes to the imager inside the prime focus cage. The design of the cooling system along with commissioning experiences and initial cooling system performance is described. The LN2 cooling system with the DES imager was initially operated at Fermilab for testing, then shipped and tested in the Blanco Coudé room. Now the imager is operating inside the prime focus cage. It is shown that the cooling performance sufficiently cools the imager in a closed loop mode, which can operate for extended time periods without maintenance or LN2 fills.
\end{abstract}

Keywords: Cryogenic, closed loop, two phase, telescope, camera, CTIO

PACS: $07.20 . \mathrm{Mc}$

\section{INTRODUCTION}

The Dark Energy Camera (DECam)[1], is the primary instrument used in the Dark Energy Survey[2]. DECam is a 3 sq. deg. mosaic camera mounted at the prime focus of the Blanco $4 \mathrm{~m}$ telescope at the Cerro Tololo Inter- American Observatory (CTIO). The camera imager[4] is a vacuum vessel that houses the CCD array, the focal plane support plate, and the liquid nitrogen heat exchanger used to cool the array[3]. The CCD array operates at $-100^{\circ} \mathrm{C}$ and has strict requirements on temperature stability and temperature uniformity across the array independent of the telescope orientation[9]. The liquid nitrogen system is a closed loop, two-phase circulation system. Liquid is pumped from the liquid nitrogen process vessel and circulated continuously to the imager vessel heat exchanger. The coolant returns back to the process reservoir where the liquid phase is separated and the gas phase is condensed using cryocoolers. The cooling strategy was determined by comparing various cooling techniques and methods used on other telescope instruments. The method best suited for an imager with such a large CCD array was determined to be a closed loop, two-phase liquid nitrogen system[6]. A prototype closed loop liquid nitrogen system was constructed in late 2008 and is used to cool a Multi-CCD test vessel[7]. A second final system was constructed with modifications from the original system to help further mitigate system thermal loads. The LN2 cooling system for the imager was then tested in late 2010 on the telescope simulator at Fermilab.[8]. In July of 2011, the LN2 cooling system was shipped and installed at CTIO. Preliminary testing was performed to ensure the cooling system was working and the control instrumentation was being data-logged to a web based system. In December of 2011, the imager was shipped to CTIO. The Imager was setup in the Coudé room at the 4 meter Blanco dome and was cooled down to test the system integration[5]. In August 2012, the telescope utility routing on the telescope and installation of the imager inside the prime focus cage took place. Initial performance of the imager cryogenic cooling system in the prime focus cage is described.

\section{DESCRIPTION OF THE LN2 CRYOGENIC COOLING SYSTEM}

The process circulation system for the cryogenic cooling is located off the telescope on the console room roof. The system has the LN2 process tank that houses the circulation pump, and the valve box. The valve box is for selecting operations on the telescope or in the Coudé room. The liquid is circulated from the process tank to the imager and 


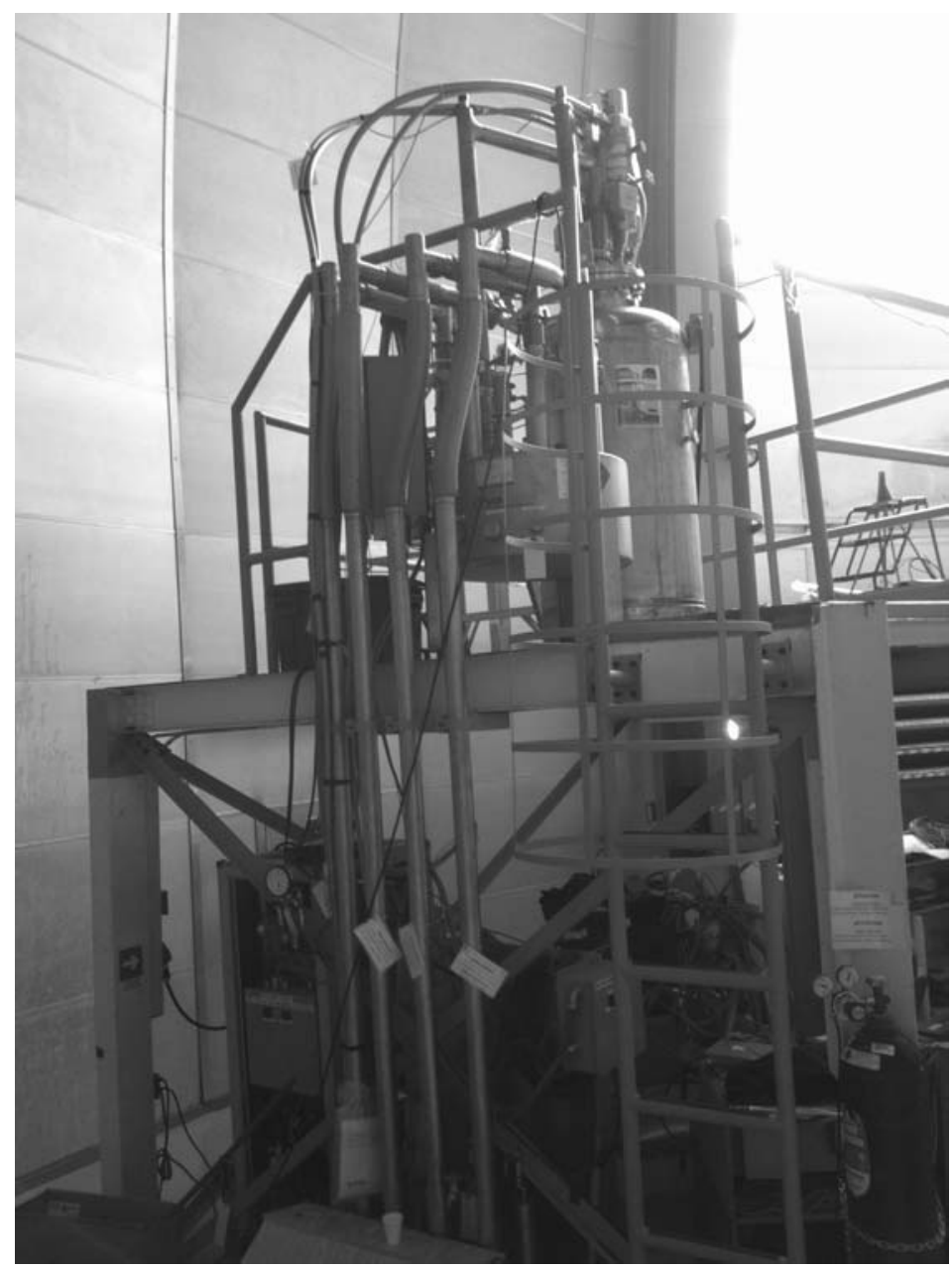

FIGURE 1. Process tank on the console room roof with valve box and transfer lines running to the Coudé room and to the telescope. The controls and instrumentation rack is located below the roof.

returns back to the process tank as a two-phase fluid where it is separated into liquid and gaseous phases. The gaseous phase is condensed using two cryocoolers. To maintain a closed loop system, the overall system heat load must be less than the cryocooler cooling capacity. The LN2 process tank installed on top of the console room is shown in Fig. 1. The process tank is the LN2 reservoir containing approximately 200L of LN2. Inside the process tank at the bottom is a circulation pump. The circulation pump is a submersible pump manufactured by Barber-Nichols. The pump is capable of a 2 gpm LN2 flow rate with a 35 psi head pressure. Mounted to the top of the process tank are two AL-300 Cryomech cryocoolers that condense returning vapor from the circulated two-phase LN2 flow. The crycocoolers with attached copper fins for condensation are shown in Fig.2. The combined cooling capacity of the two cryocoolers is a little more than 700 Watts. The overall system thermal load is less than 700 Watts, allowing the system to run in a closed loop without the need to refill with LN2. The LN2 process tank pressure is maintained at 100 psig by a control system that uses heaters inside the LN2 reservoir to vaporize additional LN2 as needed to control the pressure to 100 psig. The LN2 is circulated using the pump inside the LN2 process tank through a valve box, then to either the Coudé room or the telescope depending on where the imager is located at the time of cool down.

The LN2 vacuum jacketed transfer lines and utilities are mounted to the telescope and run to the imager in the prime focus cage. To accommodate the polar axis and declination axis motions, the transfer lines and utilities are run on the telescope in flexible cable trays for each rotating axis. The individual utilities are separated within the cable tray using tray spacers. A general layout of the piping on the telescope is shown in Fig.3. The LN2 vacuum jacketed transfer lines 

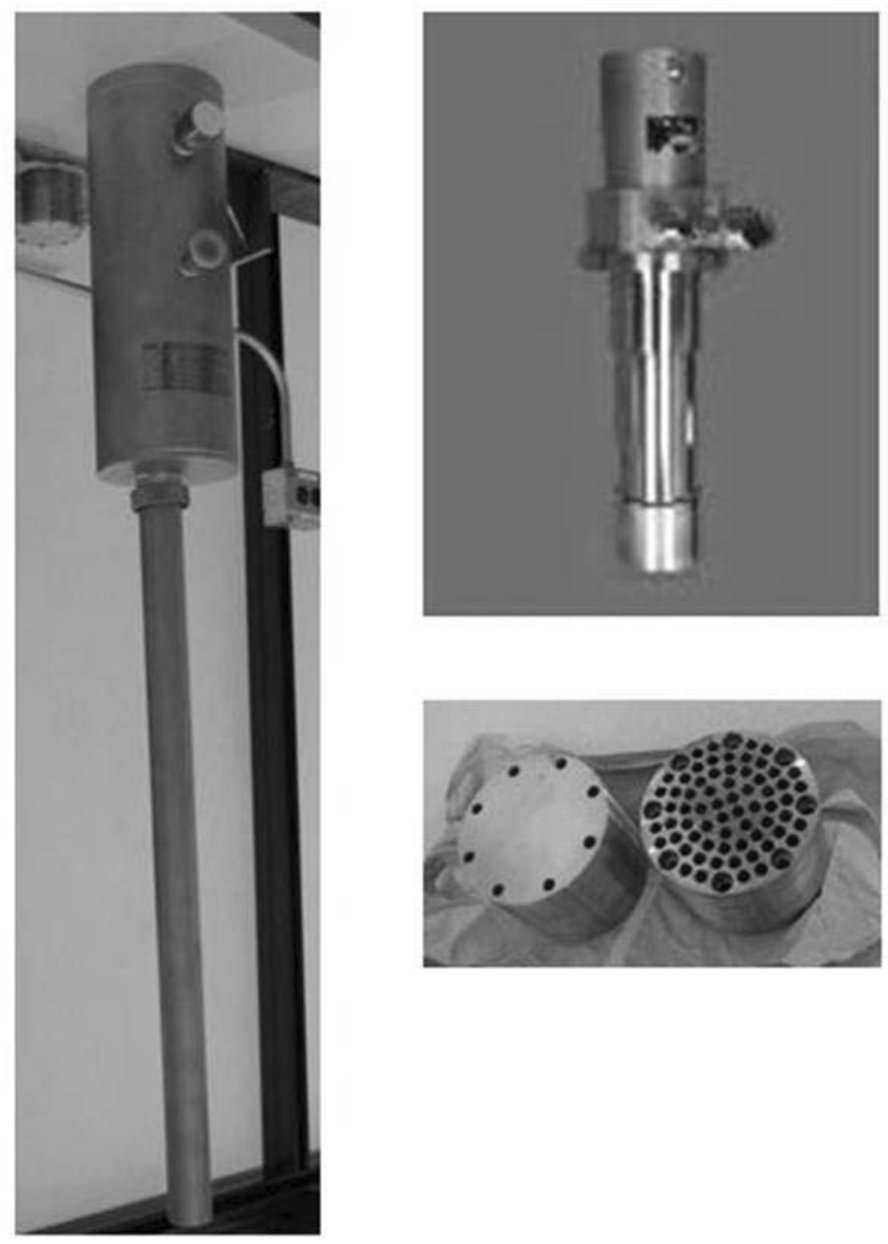

FIGURE 2. Left: Cryocooler vacuum jacketed insert. Upper right: Al-300 cryocooler. Lower right: copper fin vapor condenser.

are mounted in sections using VCR joints on the internal cooling line and a retractable vacuum bellows in the vacuum jacket for access to the VCR joint. There are seven sections of transfer lines from the valve box to the camera separated by joints. Each vacuum break joint has a vacuum pump-out port. There are about 160 feet of hard pipe combined with 75 feet of hose in each supply and return line for a total of 470 feet of piping.

The console room has the controls instrumentation rack for the cooling system and the windows computer running LabVIEW from National Instruments. The control system continuously monitors the LN2 flow rate, system pressures and also the other utilities such as the imager vacuum and water-glycol cooling systems. The control system has alarm features to warn the operator of conditions that are moving out of the normal operation parameters. Initial warnings are sent via email. As the parameters degrade and a second limit is approached, an autodialer places an automated phone call to the operator. If the parameter is severely out of range, the system goes into a self-protection mode and starts a predetermined shutdown procedure for the problem.

The prime focus cage instrumentation and utilities are routed up the telescope inside fixed and flexible section cable trays. Cage end cap and side skins are used to prevent light from reflecting off the cage components and provide a thermal barrier between the cage the the surrounding air. A cage cooling heat exchanger is used to remove any excess electronic heat once the cage skins are put on the cage. At the rear of the camera is power distribution and the camera vessel vacuum pump controllers. National Instruments control modules for the focal plate temperature and cage instrumentation is also at the end of the cage. The LN2 transfer lines, water cooling lines and other utilities are 


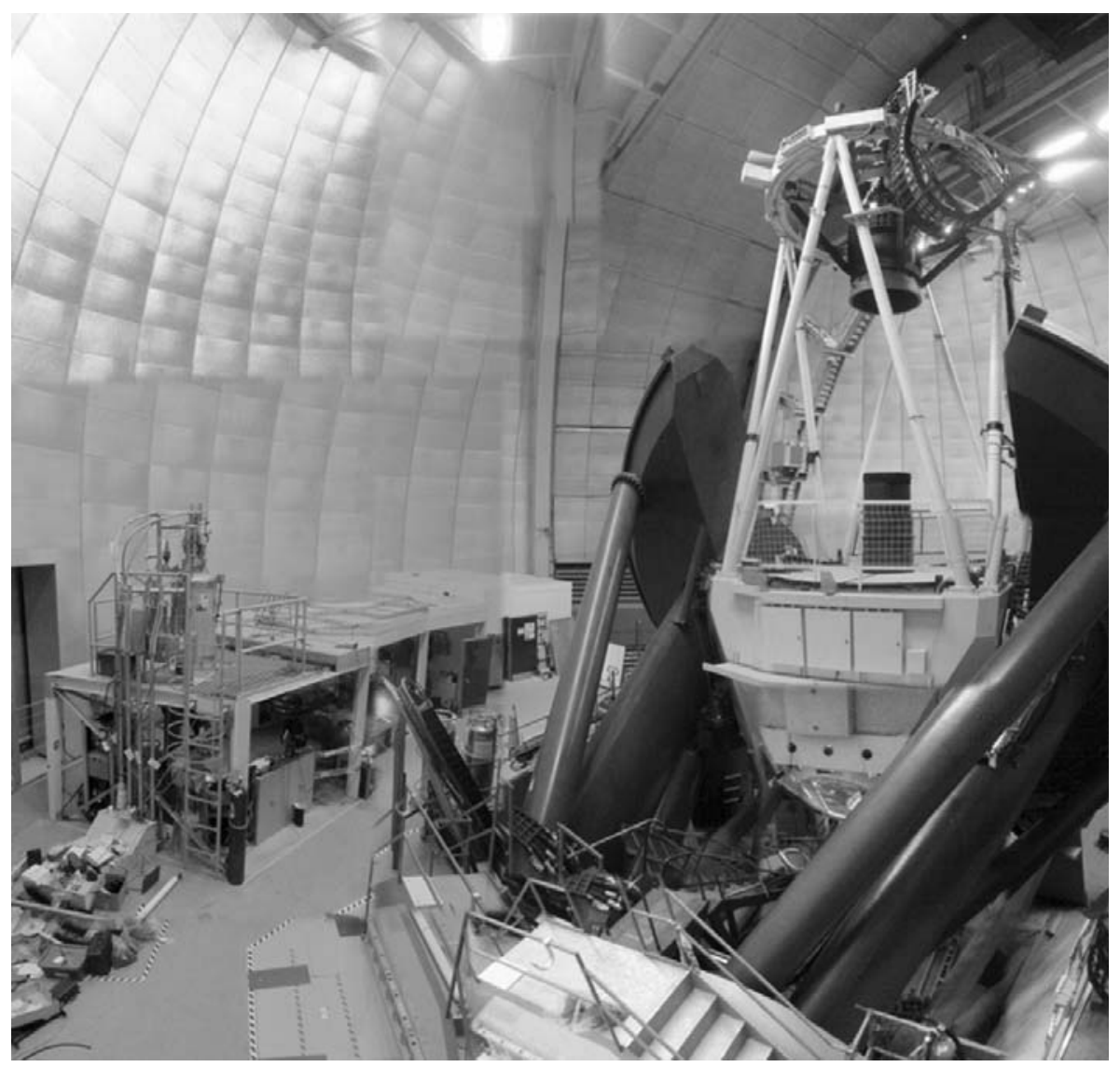

FIGURE 3. Utility routing on the telescope.

supported by the cage fin and enter the cage at the back.

\section{THE IMAGER HEAT EXCHANGER DESCRIPTION}

The heat exchanger inside the imager is a simple tube heat exchanger. Ten flexible copper straps connect the heat exchanger to the back surface of the focal plane support plate that supports the CCD array. An electric trim heater and Resistance Temperature Detector (RTD) are installed on each of the flexible copper straps. The power to each heater is modulated using PID controls to modulate the CCD array temperature. The cold sink for the focal plate is the twophase LN2 cooling fluid that is circulating in the heat exchanger. The back of the imager vessel, internal cooling ring, and copper braids are shown in Fig.4. The front of the imager with the CCD array is shown in Fig.5. Four temperature sensors at the front of the focal plate are used in combination with the braid sensors. A cascade PID loop is used to control the temperature of the focal plate using both sets of temperature sensors. The focal plate operating temperature is maintained at $-100^{\circ} \mathrm{C}$ with an expected heat load of 113 Watts including the trim heaters. The aluminum covered and insulated front end electronic crates are located on the outside of the imager. One of the four crates is used to power the trim heaters for focal plate thermal management. 

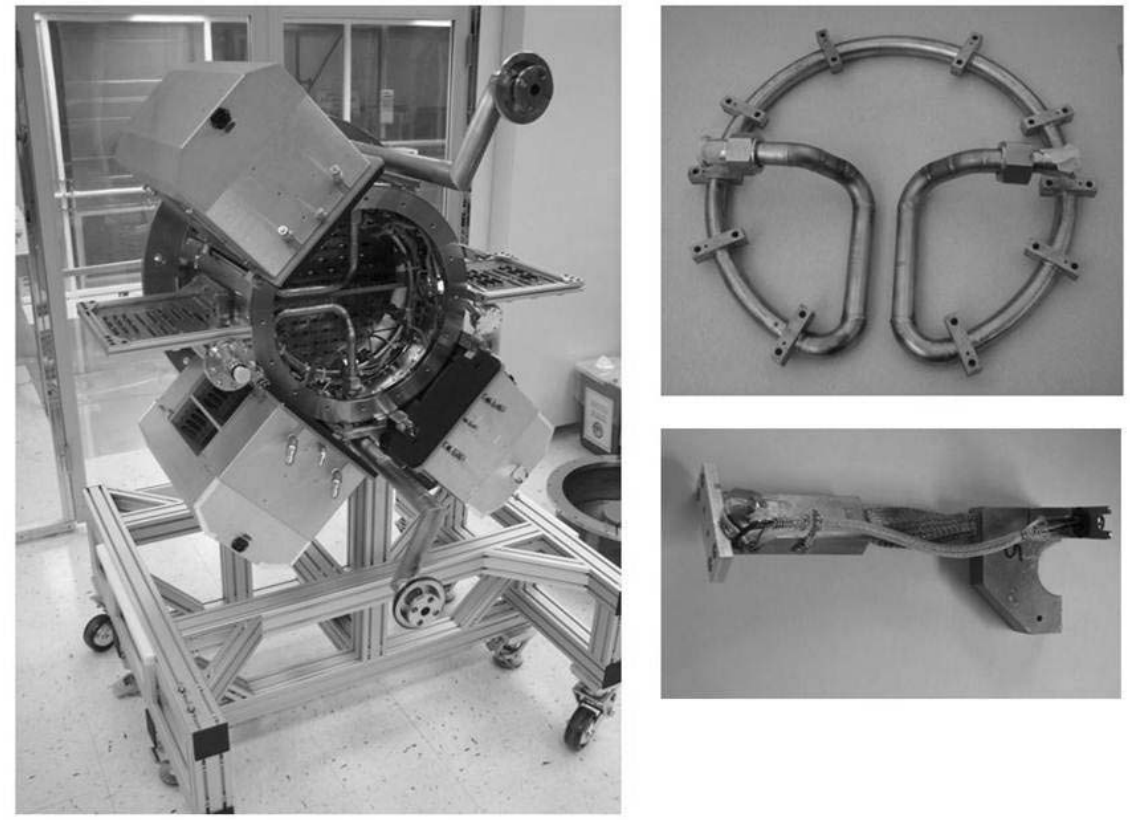

FIGURE 4. Left: Imager Vessel. Upper Right: Internal heat exchanger cooling ring. Lower Right: Copper braid with heater, temperature sensor, thermal protection switch, and electrical isolation joint.

\section{FIRST IMAGER COOL DOWN}

Previous testing of the cooling system occurred during the April 2012 cool down and warm up of the imager. The LN2 cooling system performed at CTIO with the Imager in the Coudé room as it did in early testing at Fermilab[5]. The imager is now mounted in the telescope's prime focus cage. The focal plate temperature, heat loads, and vacuum level were monitored during the cooling cycle. The data for the first cool down of the imager with it mounted in the prime focus cage is described. Each plot corresponds to the first 15 hours of the cool down.

The temperature of the focal plate next to the CCDs was monitored during the cool down procedure. The temperature, $\left({ }^{\circ} \mathrm{C}\right)$, is plotted as a function of calendar time in Fig.6. The plot shows that it takes approximately 4 hours for the imager to cool down to $-100^{\circ} \mathrm{C}$ and another few hours for the temperature control loop to stabilize the temperature to $-100^{\circ} \mathrm{C} \pm-0.25^{\circ} \mathrm{C}$. The lines are precooled by pumping $\mathrm{LN} 2$ from the supply dewar, through the supply line to the imager, back through the return line, and out a vent on the return line near the process tank. This allows the transfer lines to precool before starting the two-phase closed loop system. During cool down, the cryocoolers do not have the additional capacity for the total system heat load and the added heat load from the cool down. Once the imager is cold, it remains cold with very little regular maintenance to the LN2 cooling system. The warm up of the camera, imager focal plate, and transfer lines occur passively, without heaters, and takes approximately 24 hours to warm up. To warm up the system, the circulation pump is stopped and the LN2 is allowed to drain back into the LN2 tank on the console roof.

The focal plate temperature is maintained using a control loop and trim heaters. The power required by the trim heaters to maintain the focal plate temperature to $-100^{\circ} \mathrm{C}$ is approximately 110 Watts as shown in Fig.7. This excess cooling capability allows for faster responses to the thermal disturbance when powering on and off the CCD electronics. The additional nitrogen vapor in the cooling system created by the high capacity trim heaters is condensed by the AL-300 cryocoolers in the LN2 process tank. The extra cooling capacity at the focal plate is also used as contingency in the event that the thermal loading on the imager were different once it experiences seasonal temperature variations. Fluctuations in the power required to maintain the focal plate at the required temperature can be caused by changes in the focal plate thermal load or changes in the LN2 coolant temperature. The focal plate thermal load changes if the front end electronic crates are powered on or off. Another cause of trim heater disturbances is rapid 


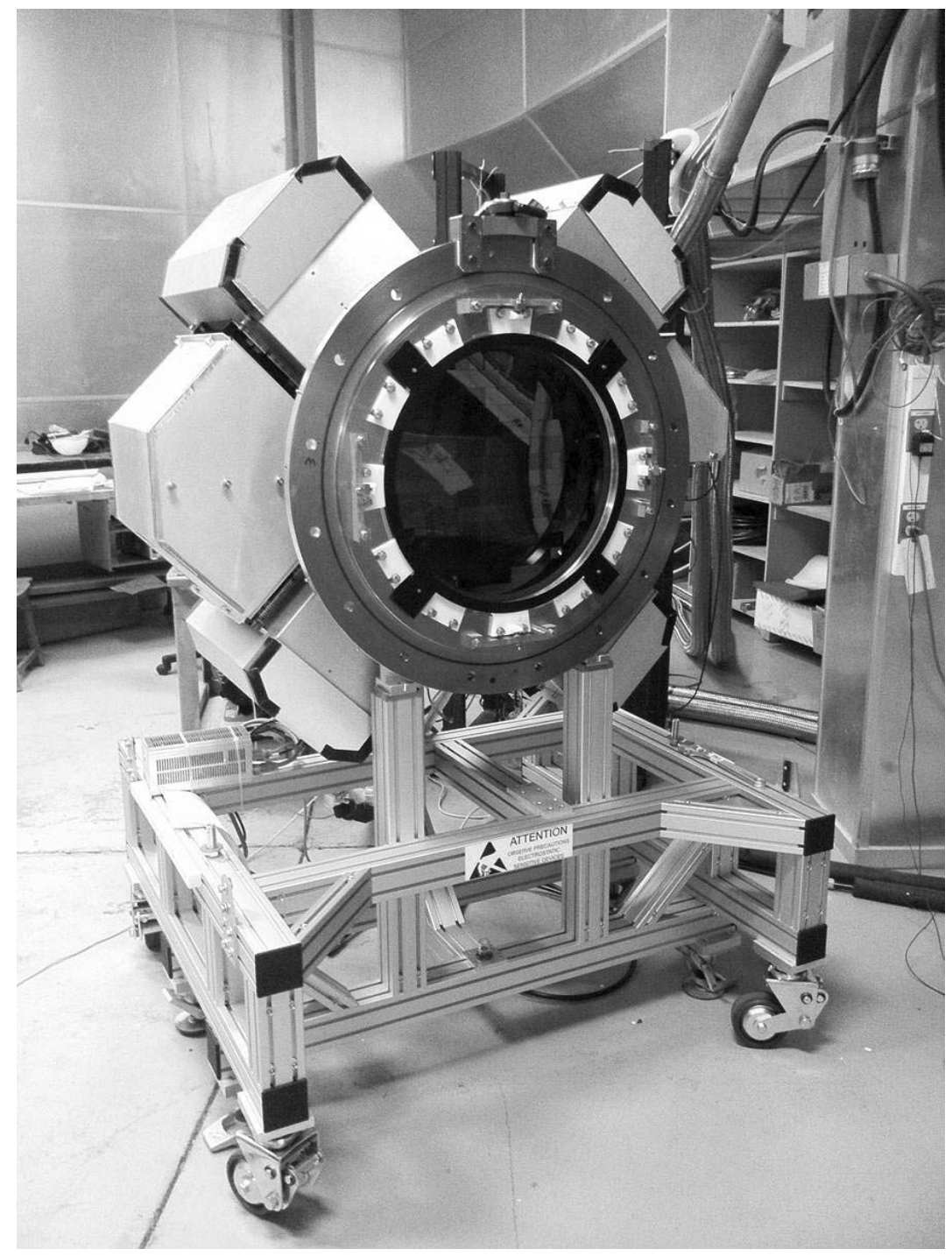

FIGURE 5. Imager front view showing the $\mathrm{C} 5$ lens, the $\mathrm{CCD}$ array, four temperature sensors at the edge of the array, Front End Electronic crates.

fluctuations in LN2 pressures during refilling the LN2 process tank (rare events).

The overall cooling system also has additional cooling capacity as shown by the heater power required in the LN2 process tank to maintain the coolant pressure to 100 psig in Fig.7. Initially, when LN2 is not being circulated, it takes approximately 700 Watts to maintain tank pressure. As the cooling system starts the cool down and liquid is circulated throughout the system, the system heat load goes up, and the heater power required to maintain the tank pressure decreases. Once the imager is cold, the figure shows approximately 100 Watts of extra cooling capacity in the system. This includes the additional 110 Watts of additional contingency at the focal plate. If the LN2 process tank heater power decreases, it is an indication that the environmental heat load on the system has increased. It is a useful diagnostic tool for monitoring the thermal load on the piping system. Weak vacuum on the transfer lines would cause an increase in system thermal load and cause additional two-phase vapor. The LN2 process tank heaters would then require less power due to the excess system thermal loading to maintain the tank at $100 \mathrm{psig}$.

The imager vacuum pressure during the cool down is shown in Fig.9. The imager volume is actively pumped for several days using a turbo pump mounted to the imager. The turbo pump can be isolated from the imager using a gate 


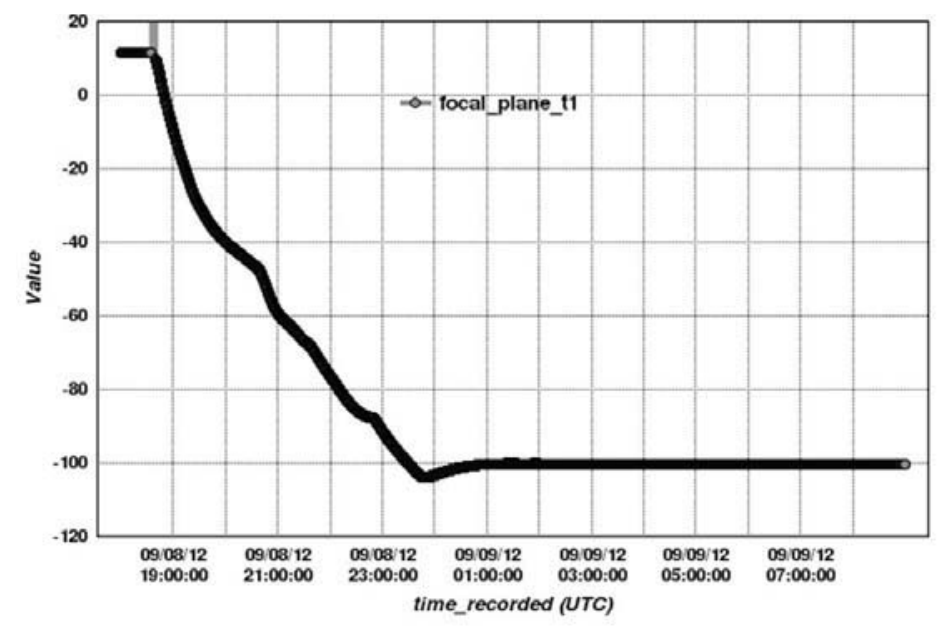

FIGURE 6. Focal plate temperature $\left({ }^{\circ} \mathrm{C}\right)$ during cool down.

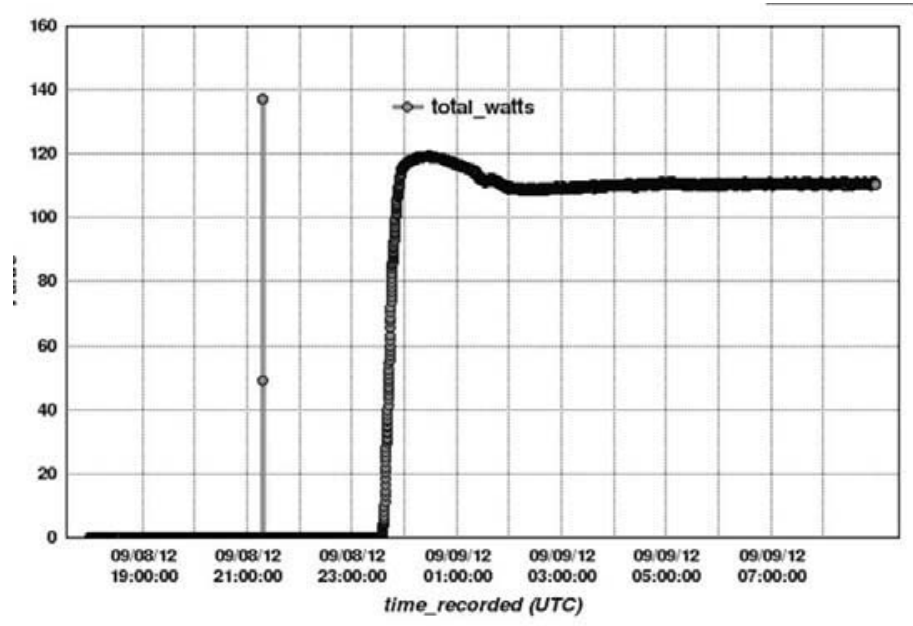

FIGURE 7. Focal plate trim heater power (Watts) required to maintain the focal plate temperature at $-100^{\circ} \mathrm{C}$.

valve for maintenance. An ion pump is mounted on the imager vessel and is used to maintain the vacuum in the event the turbo pump is not available. Both pumps and the vacuum readout gage are all electrically isolated from the imager to help minimize any electronic noise issues with the detector readout electronics. While the imager is warm, the vacuum is approximately $4 \mathrm{e}-7$ torr. Once LN2 is circulated through the imager heat exchanger, the vacuum improves to approximately 1e-7 torr due to cryopumping on the cold surfaces. During the warm-up cycle, the vacuum pressure decays due to the additional gas load coming from what was condensed on the cold LN2 heat exchanger inside the imager. It is planned for the turbo pump to run continuously with its roughing pump mounted in the cassegrain cage during the warm-up procedures.

\section{COOLING SYSTEM PERFORMANCE DURING OPERATIONS AT CTIO}

The LN2 cooling system performance is monitored during telescope motions with the cold imager mounted in the prime focus cage. The focal plate temperature, the trim heater power required to maintain that temperature and the 


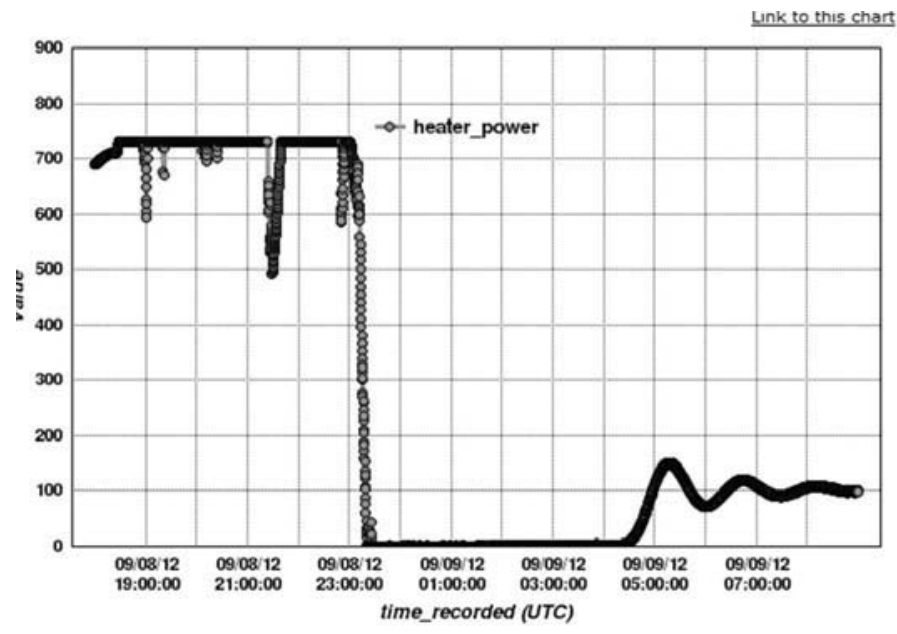

FIGURE 8. Heater power (Watts) required to maintain the LN2 process tank at the 100 psi operating setpoint.

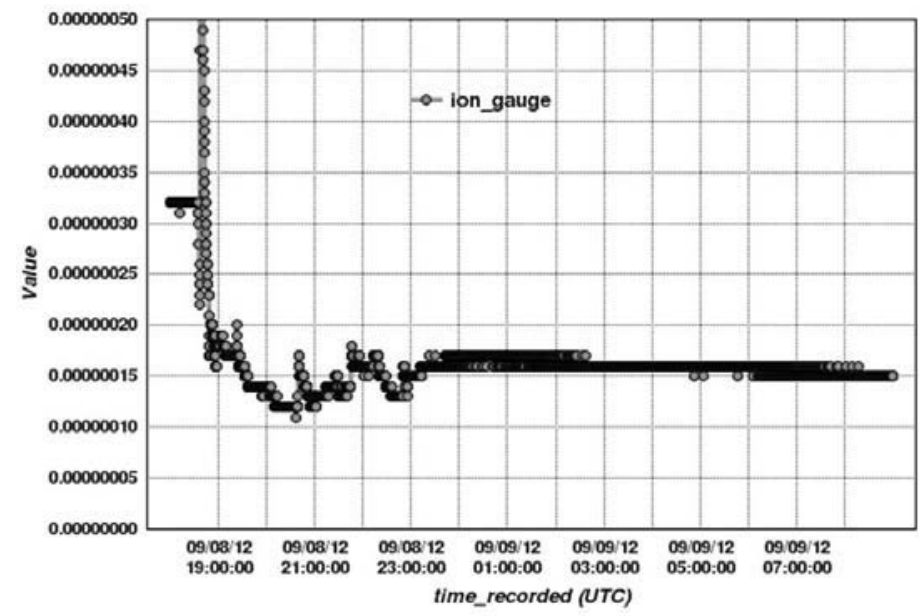

FIGURE 9. Imager vacuum (Torr) during the cool down with the turbo pump operating.

differential pressure across the LN2 circulation pump is described during an 8 hour period. The telescope orientation starts at zenith, is moved to the northwest maintenance station with the lowest elevation, then moved to the north station at a medium elevation, and back to zenith.

The differential pressure across the circulation pump increases as the imager elevation increases. The $100 \mathrm{psig}$ coolant pressure, and approximately $2 \mathrm{gpm}$ flow rate is enough to prevent the two-phase flow from separating and slowly gathering vapor bubbles in one location in the piping system. The differential pressure is shown in Fig. 10.

The temperature of the focal plate next to the CCDs is shown in Fig. 11. Even though the LN2 circulation is changing due to the differences in head pressure, the CCD temperature is maintained within $0.08{ }^{\circ} \mathrm{C}$.

The focal plate cooling strap trim heater power required to maintain the CCD temperature during changes in the flow conditions is shown in Fig.12. Small changes in the supplied heater wattage compensate for the differences in flow conditions. 


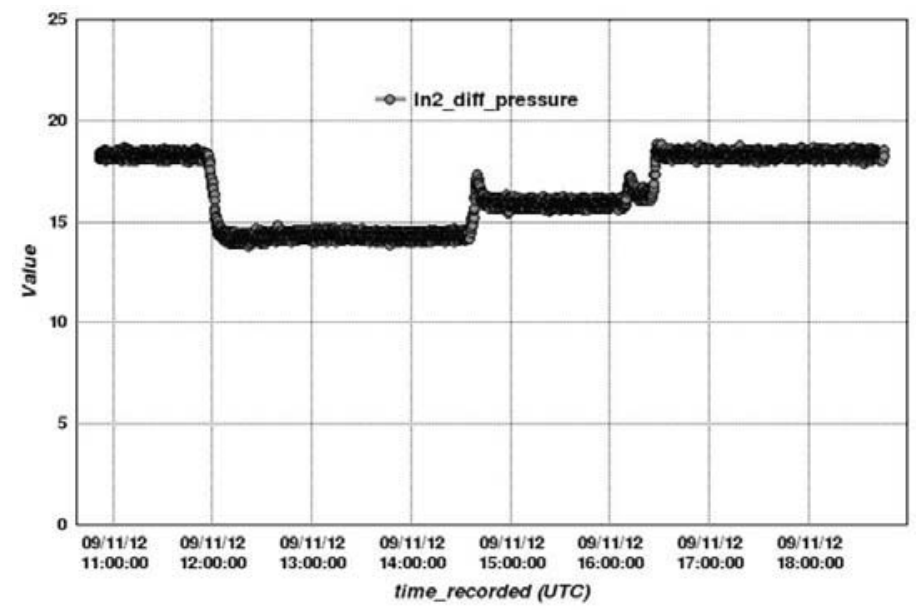

FIGURE 10. Differential pressure (psi) across the LN2 circulation pump during an 8 hour operating period with telescope motions.

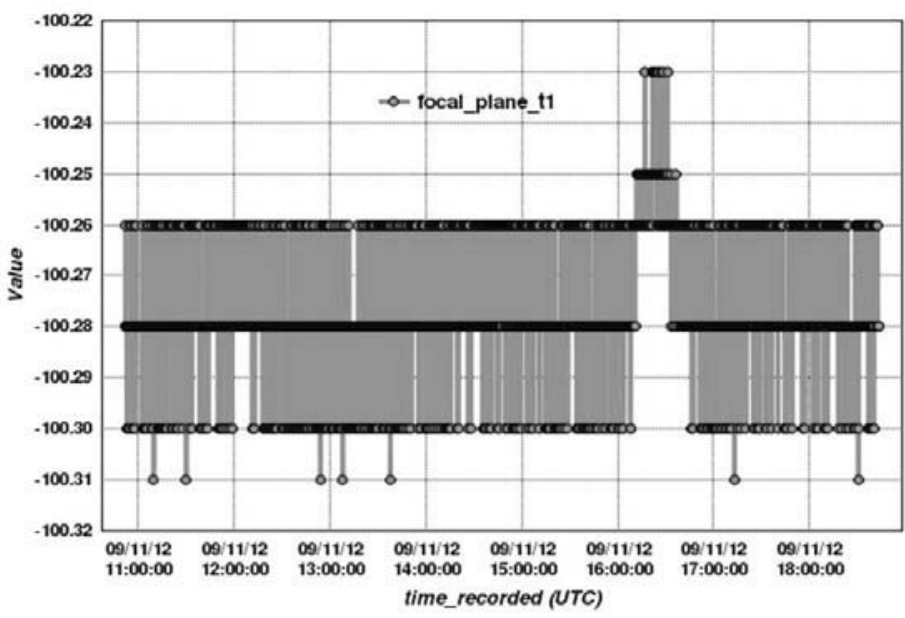

FIGURE 11. Focal plate temperature $\left({ }^{\circ} \mathrm{C}\right)$ during an 8 hour operating period with telescope motions.

\section{SUMMARY}

The LN2 cooling system with the DES imager was initially operated at Fermilab for testing, then shipped to Chile and tested in the Blanco Coudé room at CTIO. At present, the imager is operating inside the telescope's prime focus cage. The cooling performance sufficiently cools the imager in a closed loop mode, which can operate for extended time periods (months) without additional LN2 fills. The cool down time is 4 hours before the CCDs can be read, and another few hours before the temperature is stable within the requirements of $-100^{\circ} \mathrm{C}+/-0.25^{\circ} \mathrm{C}$. The warm up of the imager is passive, which allows the transfer lines to warm up passively as well. The imager vacuum is maintained actively either by an ion pump or a turbo pump with the roughing pump mounted remotely in the cassegrain cage. 


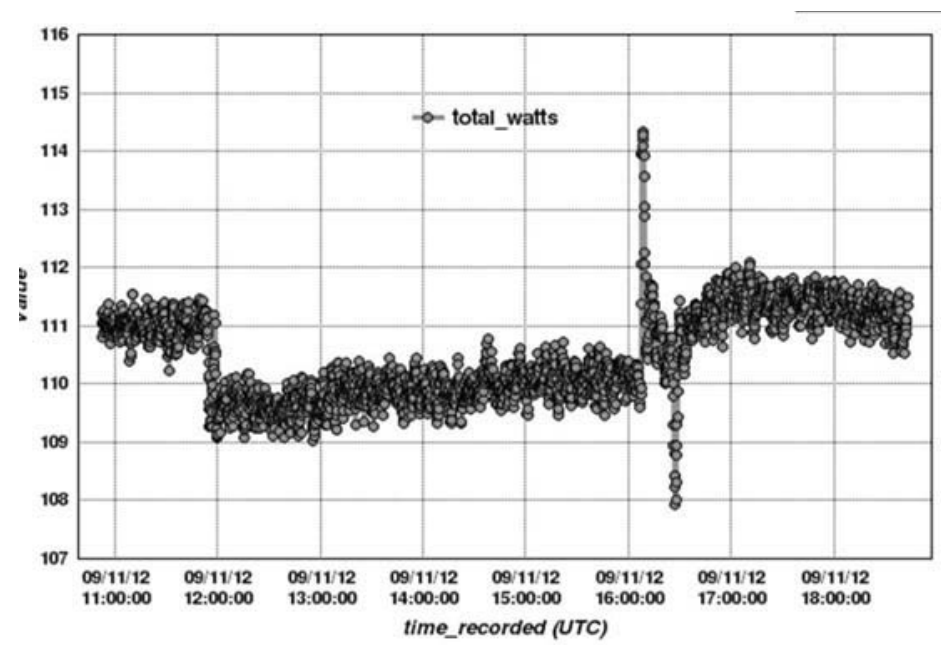

FIGURE 12. Trim heater power (Watts) during an 8 hour operating period with telescope motions.

\section{ACKNOWLEDGMENTS}

Funding for the DES Projects has been provided by the U.S. Department of Energy, the U.S. National Science Foundation, the Ministry of Science and Education of Spain, the Science and Technology Facilities Council of the United Kingdom, the Higher Education Funding Council for England, the National Center for Supercomputing Applications at the University of Illinois at Urbana-Champaign, the Kavli Institute of Cosmological Physics at the University of Chicago, Financiadora de Estudos e Projetos, Fundação Carlos Chagas Filho de Amparo à Pesquisa do Estado do Rio de Janeiro, Conselho Nacional de Desenvolvimento Científico e Tecnológico and the Ministério da Ciência e Tecnologia, the Deutsche Forschungsgemeinschaft and the Collaborating Institutions in the Dark Energy Survey.

The Collaborating Institutions are Argonne National Laboratories, the University of California at Santa Cruz, the University of Cambridge, Centro de Investigaciones Energeticas, Medioambientales y Tecnologicas-Madrid, the University of Chicago, University College London, DES-Brazil, Fermilab, the University of Edinburgh, the University of Illinois at Urbana-Champaign, the Institut de Ciencies de l'Espai (IEEC/CSIC), the Institut de Fisica d'Altes Energies, the Lawrence Berkeley National Laboratory, the Ludwig-Maximilians Universität and the associated Excellence Cluster Universe, the University of Michigan, the National Optical Astronomy Observatory, the University of Nottingham, the Ohio State University, the University of Pennsylvania, the University of Portsmouth, SLAC, Stanford University, the University of Sussex, and Texas A\&M University.

\section{REFERENCES}

1. Flaugher, B.“The Dark Energy Survey”, Int. J. Modern. Phys. A20, 3121 (2005)

2. Flaugher, B. et al.,"Status of the Dark Energy Survey Camera (DECam) Project" Proc. SPIE, Volume 7735 (2010)

3. Derylo, et al., "Assembly of the Dark Energy Survey CCD imager", Proc. SPIE, Volume 7735 (2010)

4. Cease, H. et al., "The Dark Energy Survey CCD imager design", Proc. SPIE, Volume 7014 (2008).

5. Cease, H. et al., "Commissioning and Initial Performance of the Dark Energy Camera Liquid Nitrogen Cooling System", Proc. SPIE, Volume 8446 (2012).

6. Schmitt, R.L. et al., "Cooling the Dark Energy Camera Instrument”, Proc. SPIE, Volume 7014 (2008).

7. Cease, H. et al., "Cooling the Dark Energy Camera CCD array using a closed loop two-phase liquid nitrogen system", Proc. SPIE, Volume 7739 (2010)

8. Diehl, H.T. et al., "Testing the Dark Energy Camera on a Telescope Simulator”, Proc. SPIE, Volume 7735 (2010)

9. Abbott, T., Annis, J., DePoy, D.L., Flaugher, B., Kent, S., Lin, H., Merritt, W., "Dark Energy Camera Specifications and Technical Requirements", 24 August 2012 